Supporting Information:

\title{
Rapid and Robust Coating Method to Render Polydimethylsiloxane Surfaces Cell Adhesive
}

\author{
David B. Gehlen, ${ }^{1}$ Leticia C. De Lencastre Novaes, ${ }^{1}$ Wei Long, ${ }^{2}$ Anna Joelle Ruff, ${ }^{2}$ Felix Jakob, ${ }^{2}$ \\ Tamás Haraszti, ${ }^{1}$ Yashoda Chandorkar, ${ }^{1}$ Liangliang Yang, ${ }^{3}$ Patrick van Rijn, ${ }^{3}$ Ulrich Schwaneberg, ${ }^{2}$ \\ and Laura De Laporte ${ }^{1,4^{*}}$
}

${ }^{1}$ DWI - Leibniz Institute for Interactive Materials, Forckenbeckstraße 50, D-52074 Aachen, Germany

${ }^{2}$ Institute of Biotechnology, RWTH Aachen University, Worringerweg 3, D-52074 Aachen, Germany

3University Medical Center Groningen, Department of Biomedical Engineering, FB40, 9713 AV Groningen, The Netherlands

${ }^{4}$ Institute for Technical and Macromolecular Chemistry, RWTH Aachen University, Worringerweg 1-2, D-52074 Aachen, Germany

E-mail: delaporte@dwi.rwth-aachen.de 


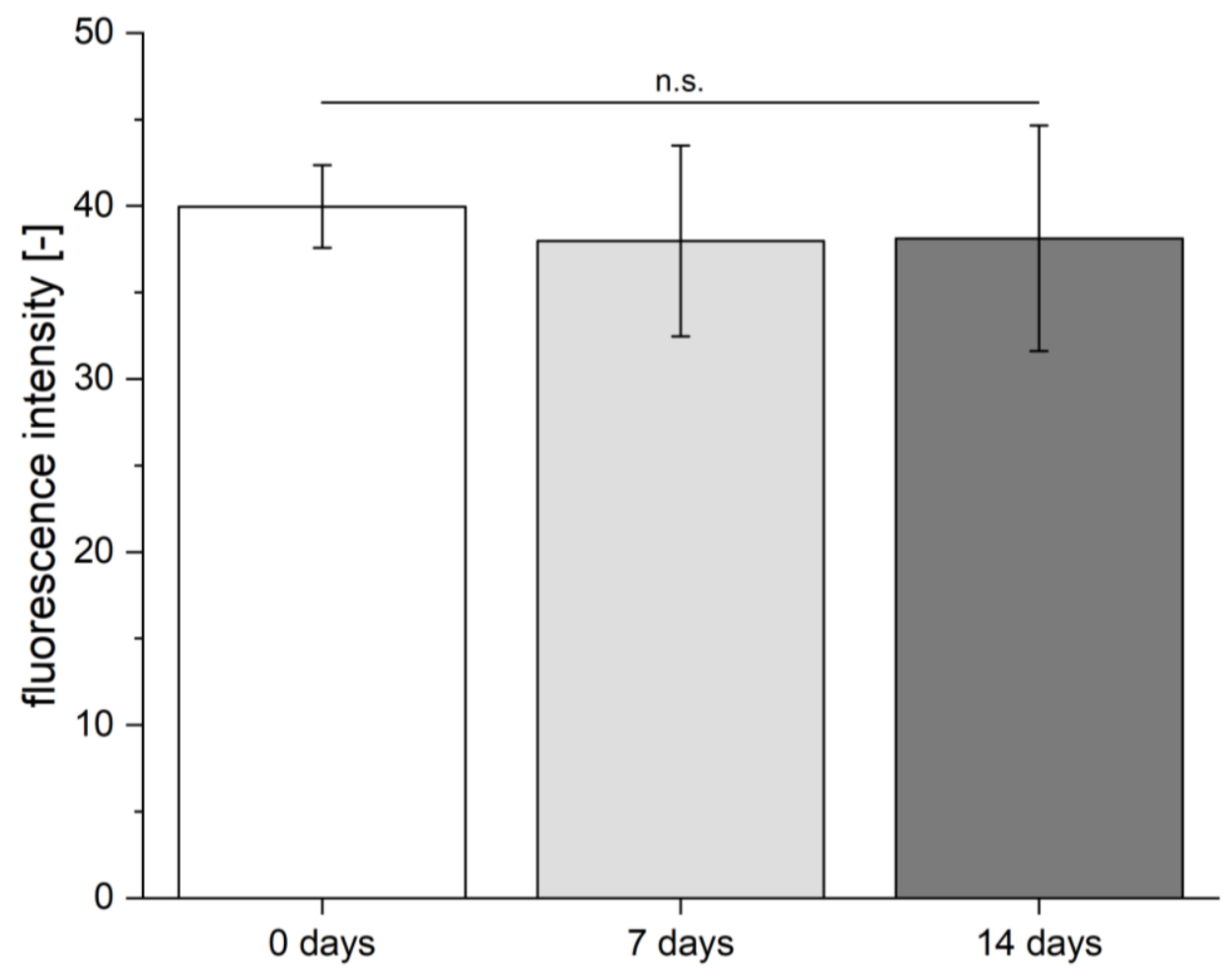

Figure S1: Long-term stability of eGFP-coatings on PDMS in PBS for different time points revealing no changes in the fluorescence intensity / stability after 14 days. 


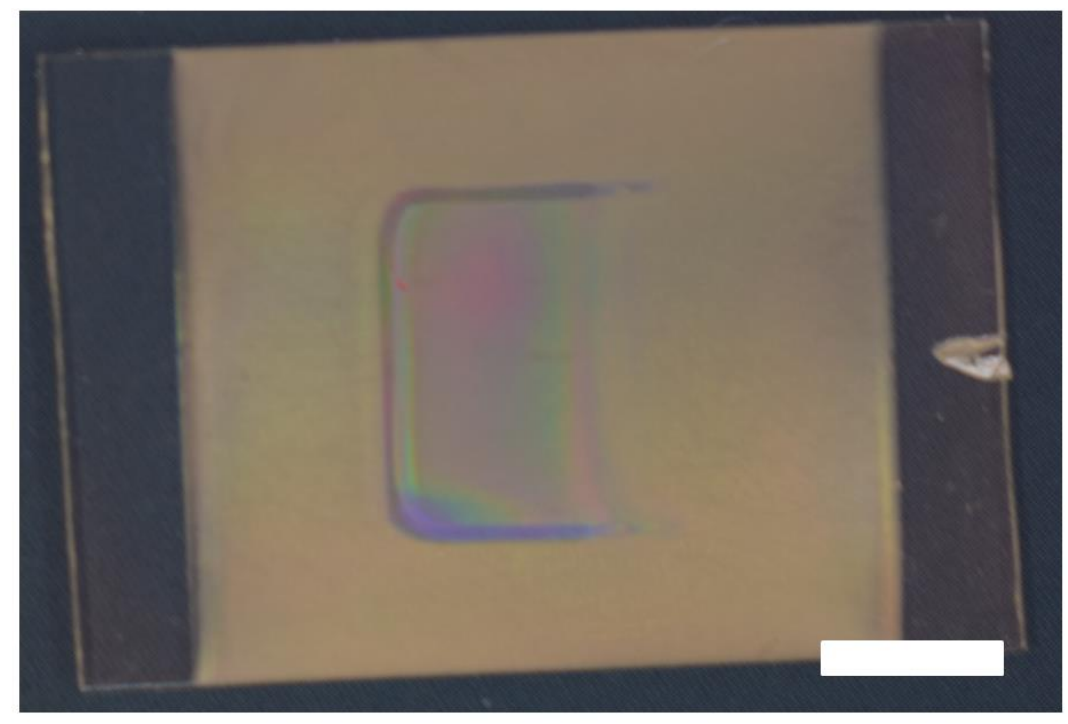

Figure S2: PDMS based directional nanotopographic gradient over a size of $10 \times 10 \mathrm{~mm}$, showing different light scattering depending on the wrinkle size. Scale bar represents $5 \mathrm{~mm}$. 